Research Paper

\title{
The Myosin II Inhibitor, Blebbistatin, Ameliorates FeCl3-induced Arterial Thrombosis via the GSK3ß-NF-KB Pathway
}

\author{
Yuanyuan Zhang, Long Li, Yazheng Zhao, Han Han, Yang Hu, Di Liang, Boyang Yu, Junping Kou \\ Jiangsu Key Laboratory of TCM Evaluation and Translational Research, Department of Complex Prescription of TCM, China Pharmaceutical University, 639 \\ Longmian Road, Nanjing 211198, China. \\ $\triangle$ Corresponding author: Dr Junping Kou, Jiangsu Key Laboratory of TCM Evaluation and Translational Research, Department of Complex Prescription of \\ TCM, China Pharmaceutical University, 639 Longmian Road, Nanjing 211198, China Tel\&Fax: +86- 25- 86185158 E-mail: junpingkou@cpu.edu.cn \\ (c) Ivyspring International Publisher. This is an open access article distributed under the terms of the Creative Commons Attribution (CC BY-NC) license \\ (https://creativecommons.org/licenses/by-nc/4.0/). See http://ivyspring.com/terms for full terms and conditions.
}

Received: 2016.11.24; Accepted: 2017.03.07; Published: 2017.05.15

\begin{abstract}
Arterial thrombosis and its related diseases are major healthcare problems worldwide. Blebbistatin is an inhibitor of myosin II, which plays an important role in thrombosis. The aim of our study is to explore the effect and potential mechanism of blebbistatin on arterial thrombosis. A ferric chloride $\left(\mathrm{FeCl}_{3}\right)$ solution at a concentration of $5 \%$ was used to induce carotid artery thrombosis in mice. Immunohistochemistry and immunofluorescence staining were used to detect the expression or activation of non-muscle myosin heavy chain IIA (NMMHC IIA), tissue factor (TF), GSK3 $\beta$ and NF-KB. Blebbistatin ( $1 \mathrm{mg} / \mathrm{kg}$, i.p.) significantly reduced carotid artery thrombosis induced by $\mathrm{FeCl}_{3}$ solution in mice, inhibited NMMHC IIA expression and impeded TF expression via the GSK3 $\beta-N F-K B$ signalling pathway in mouse arterial vascular tissues. The present study demonstrates that blebbistatin may impede TF expression partly via the Akt/GSK3 $\beta-N F-K B$ signalling pathways in the endothelium in a $\mathrm{FeCl}_{3}$ model, shedding new insights into the pathogenesis of arterial thrombosis and providing new clues for the development of antithrombotic drugs.
\end{abstract}

Key words: Carotid artery thrombosis, Blebbistatin, NMMHC II, Tissue factor, Endothelium.

\section{Introduction}

Arterial thrombosis (AT) is one of the major healthcare problems worldwide. It is a proximate cause of death with a high incidence rate, high morbidity and high mortality $[1,2]$. AT is generally caused by the rupture of an atherosclerotic plaque, trauma or other incidents that lead to blood clotting at the damaged part of the artery surface in the cardiocerebral vascular system. This insoluble blood clot becomes a thrombus [3]. AT generally leads to myocardial infarction, ischaemic stroke, acute superior mesenteric artery embolism and cerebrovascular diseases, which are difficult issues in clinical diagnosis and treatment [4]. These conditions occur at a high incidence rate and exhibit a high mortality rate [5-7]. There are still no safe and reliable drug therapies for the treatment of arterial thrombosis at present.

Atherosclerosis is the primary cause of arterial thrombosis, and endothelial dysfunction plays an important role in the formation of atherosclerosis and thrombosis $[8,9]$. TF is a $47-\mathrm{kD}$ membrane-bound glycoprotein that functions as the primary initiator of the coagulation cascade in haemostasis and pathological thrombosis. TF is also an essential cofactor of activated coagulation factor VIIa, which forms a complex that activates the entire blood coagulation cascade [10]. Various endogenous and exogenous agonists (e.g., tumour necrosis factor-a, TNF- $\alpha$ and lipopolysaccharides, LPS) induce TF expression in monocytes, neutrophils and endothelial cells, which initiates life-threatening thrombosis in numerous diseases, such as dispersion of 
intravascular coagulation (DIC), sepsis, atherosclerosis and tumours[11, 12]. Regulation of the TF pathway is an effective strategy for the prevention of arterial thrombosis [13].

On the other hand, non-muscle myosin heavy chain IIA (NMMHC IIA) is a member of the non-muscle myosin II (NM II) super family [14]. Recent studies have demonstrated that NMMHC IIA plays an important role in the regulation of thrombosis [15]. NMMHC IIA deficiency reduces platelet aggregation and arterial thrombosis formation [16, 17]. Our previous study suggested that the NMMHCIIA non-specific inhibitor blebbistatin inhibited NMMHC IIA, which reduced TF expression via the Akt/GSK3 $\beta$-NF- $\mathrm{kB}$ signalling pathways in the endothelium in vitro and inhibited deep vein thrombosis formation in vivo [18]. However, whether blebbistatin inhibits arterial thrombosis and modulates TF-related signalling pathways in arterial vascular tissues is still unknown. Therefore, in the present study, we observed the effect of blebbistatin on carotid artery thrombosis and expression of NMMHC IIA and TF in mice treated with a $5 \% \mathrm{FeCl}_{3}$ solution and explored the role of GSK3 $\beta-N F-K B$ signalling pathway to further elucidate the function of NMMHC IIA in thrombosis.

\section{Methods and Materials}

\section{Animals}

Male C57BL/6J mice (22-25 g) were purchased from the Model Animal Research Centre of Yangzhou University (Yangzhou, Jiangsu, China). The animals were housed in a standard vivarium with free access to food and water that was temperature $\left(23 \pm 1{ }^{\circ} \mathrm{C}\right)$, humidity $(30 \%-40 \%)$ and light controlled (in $12 \mathrm{~h}$ light/dark cycle). All animal welfare and experimental procedures were in accordance with National Institutes of Health Guide for the Care and Use of Laboratory Animals, and the protocols used were approved by the Animal Ethics Committee of China Pharmaceutical University, China Pharmaceutical University, Nanjing, China.

\section{Reagents}

Blebbistatin was purchased from Sigma Aldrich (St. Louis, MO, USA). $\mathrm{FeCl}_{3}$ was obtained from Sinopharm Chemical Reagent Co., Ltd. (Shanghai, China). An antibody against mouse TF was purchased from R\&D Systems (Minneapolis, MN, USA). Antibodies against myosin IIA, p65, phospho-p65, GSK3 $\beta$, and phospho-GSK3 $\beta$ were obtained from Cell Signalling Technology (Boston, MA, USA). Pimonidazole and 4',6-diamidino-2-phenylindole (DAPI) were purchased from Beyotime Biotechnology
(Shanghai, China). Alexa Fluor ${ }^{\circledR} 488$ Donkey Anti-Goat IgG $(\mathrm{H}+\mathrm{L})$ and Alexa Fluor ${ }^{\circledR} 594$ Donkey Anti-Goat IgG $(\mathrm{H}+\mathrm{L})$ antibodies were purchased from Invitrogen (Carlsbad, CA, USA). All other regents used in this study were of the highest purity commercially available.

\section{Carotid artery thrombosis (CAT)}

The mouse $\mathrm{FeCl}_{3}$ thrombosis model was modified based on previous reports [19, 20]. Briefly, mice were anaesthetized by intraperitoneal administration of urethane (final concentration: 5\%, $0.1 \mathrm{ml} / 10 \mathrm{~g}$ ), and a lateral tail vein was cannulated for test compound or saline (SAL) vehicle infusion. Body temperature was monitored and maintained at $37^{\circ} \mathrm{C}$ using a rectal thermometer, heat lamp and temperature control unit. A tracheostomy was performed, the right carotid artery was isolated, and a 1.3-mm Doppler flow probe was placed on the artery to measure blood flow VEL. Mean arterial blood pressure, HR and VEL data were collected using the Dataquest A.R.T.k 2.1 data acquisition program (Moor, UK). A 3-mm square patch of Whatman \#1 filter paper pre-soaked in $5 \% \mathrm{FeCl}_{3}$ in $0.9 \%$ saline solution (J.T. Baker, Phillipsburg, NJ) was placed on the right carotid artery anterior to the Doppler flow probe. Mean arterial pressure, HR and VEL were continuously monitored for 60 min following $\mathrm{FeCl}_{3}$. A terminal blood sample was drawn from the abdominal aorta $60 \mathrm{~min}$ after $\mathrm{FeCl}_{3}$ application into a syringe pre-filled with sodium citrate. Blebbistatin (1 $\mathrm{mg} / \mathrm{kg}$ body weight) or $5 \%$ ethanol in normal saline were administered i.p. $30 \mathrm{~min}$ before CAT.

\section{Haematoxylin and eosin (HE) staining}

HE staining was performed following a standard procedure. Excised vessels were dehydrated with $40 \%$ sucrose, embedded in OTC, and frozen at $-70{ }^{\circ} \mathrm{C}$. Vessels were sectioned into $10-\mu \mathrm{m}$ thick slices using a cryotome (Leica, Mannheim, Germany). Specimens were washed in PBS and stained with H\&E staining. A minimum of 10 microscopic fields from each slide was randomly selected for observation under a microscope (Carl Zeiss, Germany).

\section{Immunohistochemistry (IHC)}

IHC was performed following a standard procedure. Briefly, sections were rinsed with PBS for 5 min and incubated with a primary antibody for 60 $\mathrm{min}$ at RT. Sections were rinsed twice in PBS for $5 \mathrm{~min}$ each and incubated with a biotin-conjugated secondary antibody at $20 \sim 37{ }^{\circ} \mathrm{C}$ for $20 \mathrm{~min}$. The sections were rinsed twice in PBS for 5 min each and incubated with $\mathrm{SABC}$ reagent at $37^{\circ} \mathrm{C}$ for $20 \mathrm{~min}$. Sections were rinsed 4 times in PBS for 5 min each and 
processed using a DAB kit(Beyotime, Jiangsu, China). The degree of staining was controlled using regular microscopy. Sections were washed in distilled water and stained in haematoxylin. The sections were dehydrated and mounted on slides. The slides were observed under a fluorescence microscope (Zeiss, Germany).

\section{Immunofluorescence assay}

Sections for immunofluorescence staining were incubated with primary antibodies against CD31, TF, myosin IIA, p65, phospho-p65, GSK3 $\beta$, and phospho-GSK3 $\beta$ at $4{ }^{\circ} \mathrm{C}$ overnight. Alexa Fluor ${ }^{\circledR}$ 488-conjugated Donkey Anti-Goat IgG (H+L) (1:600) and Alexa Fluor ${ }^{\circledR}$ 594-conjugated Donkey Anti-Goat IgG $(\mathrm{H}+\mathrm{L})$ antibodies (1:800) were used as secondary antibodies (Invitrogen, Carlsbad, CA, USA). Nuclei were stained using DAPI. The slides were observed under a confocal microscope (Zeiss, Germany).

\section{Measurement of TF procoagulant activity}

The procoagulant activities of $\mathrm{TF}$ in mouse plasma were measured using a chromogenic assay as previously reported [21]. Mouse plasma was prepared via centrifugation at $3,000 \times \mathrm{g}$ for $15 \mathrm{~min}$ and stored at $-70{ }^{\circ} \mathrm{C}$ prior to use. Briefly, samples $(45 \mu \mathrm{l})$ were incubated with a reagent mixture (5 $\mu \mathrm{l}, \mathrm{pH}$ 7.3) containing a $10 \mathrm{~g} / 1$ prothrombin complex and 100 $\mathrm{mM} \mathrm{CaCl}_{2}$ in a 96-well plate. A factor Xa chromogenic substrate $(0.5 \mathrm{mM}, 50 \mu \mathrm{l})$ containing $100 \mathrm{mM}$ EDTA $(\mathrm{pH} 8.4)$ was added after incubation at $37^{\circ} \mathrm{C}$ for 15 min. The reaction was incubated at $37^{\circ} \mathrm{C}$ for $5 \mathrm{~min}$, and the absorbance was measured at $405 \mathrm{~nm}$. TF activity of the model group was set as $100 \%$.

\section{Statistical analysis}

Statistical analysis was performed using SPSS 11.0 software. All data were expressed as the means \pm SEM from at least three independent experiments. ANOVA test was used for comparison between groups. $\quad P<0.05$ was considered statistically significant.
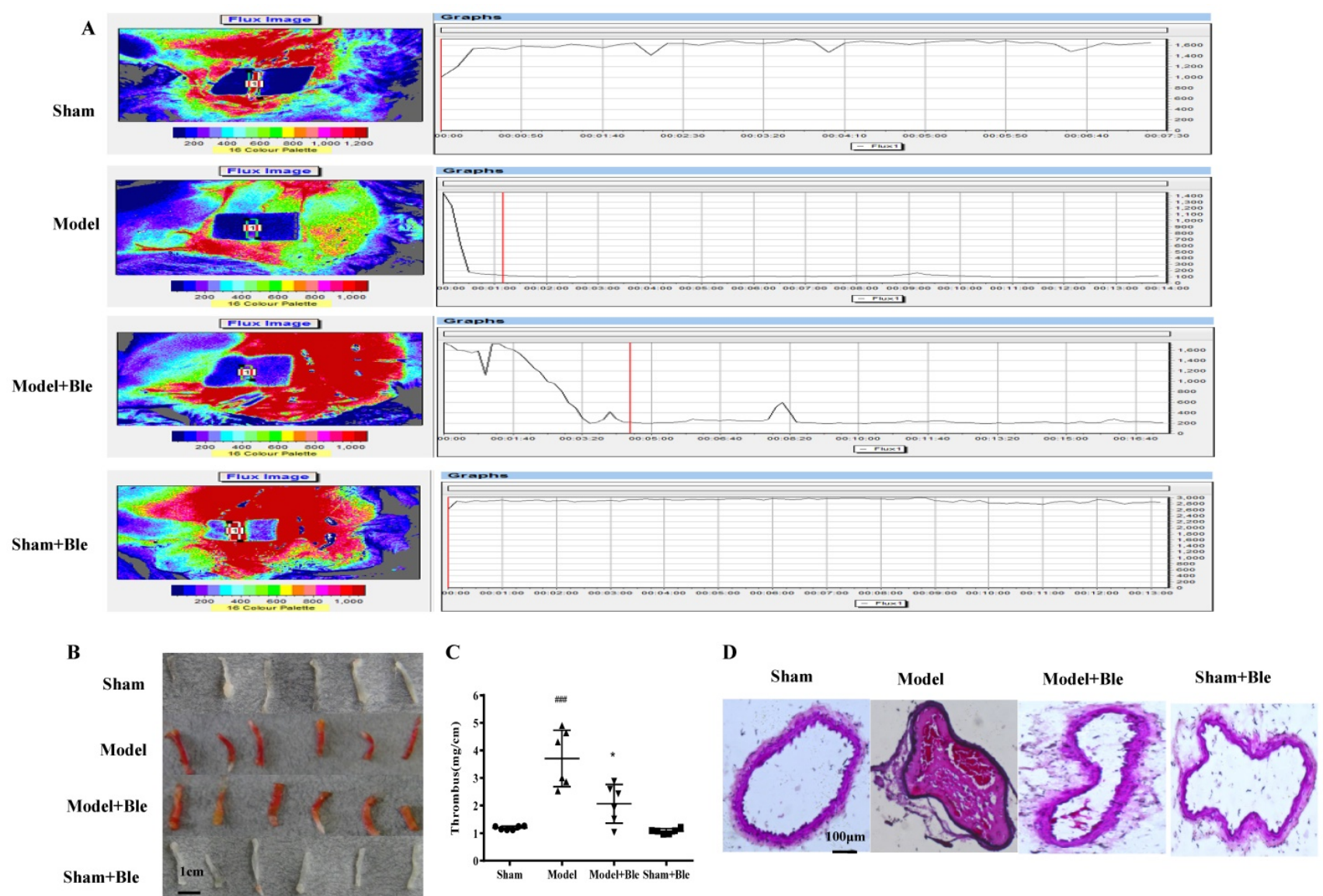

Figure 1. Blebbistatin suppresses FeCl3-induced arterial thrombosis formation. C57BL/6) mice (male, $22-25 \mathrm{~g}$ ) were injected with vehicle (5\% ethanol in saline) or blebbistatin (1 $\mathrm{mg} / \mathrm{kg}$ ) intraperitoneally $30 \mathrm{~min}$ after surgery. Mice were anaesthetizedwith a $4 \%$ chloral hydrate solution, the right carotid artery was exposed, and filter paper soaked in a $5 \%$ ferric chloride solution was placed on the surface of the carotid artery. (A). Blood flow was recorded using a colour Laser Doppler Image scanner (Moor LDI, Moor Instruments). Blood flow was monitored continuously from the onset of injury until stable occlusion occurred (defined as flow $\leq 200 \mathrm{PU}$ for $\geq 10 \mathrm{~min}$ ) or for $30 \mathrm{~min}$ if occlusion did not occur. (B)\&(C). The thrombosed carotid artery was photographed and weighed, and the length of the thrombus was measured. The size of the thrombus was quantified as $\mathrm{mg} / \mathrm{cm}$. (D). Sample $H$-E staining micrographs from mouse carotid arteries. Data represent means \pm SEM $(n=6)$. \#\#P<0.001 vs. Sham group; $* P<0.05$ vs. Model group. $(n=6)$ Ble is the shorthand versions of $B l e b b i s t a t i n$. 


\section{Results}

\section{Blebbistatin suppressed $\mathrm{FeCl}_{3}$-induced arterial thrombosis in mice}

As shown in Fig. 1A, an obvious change of blood flow was observed in the model group compared to the sham group. Carotid artery blood flow in $2 \mathrm{~min}$ was less than $200 \mathrm{PU}$ and stable in the model group. Blebbistatin $(1 \mathrm{mg} / \mathrm{kg})$ improved the blood flow value of the carotid artery compared to the model group. The results indicated that thrombosis, arterial wall thickening and agate red thrombus formation were visible in the model group induced by $5 \% \mathrm{FeCl}_{3}$ solution, while the blebbistatin-treated group exhibited alleviated thrombosis and internal carotid lumen-filling material. Moreover, the length of thrombosis in the ferric chloride group was 2.69 times longer than that in the sham group $(P<0.05)$. Blebbistatin $(1 \mathrm{mg} / \mathrm{kg})$ significantly inhibited the formation of carotid artery thrombosis with an inhibition rate of approximately $34 \%$ with significant difference. Intravascular thrombosis was not obvious in this group. The average size of the thrombus covered $89.63 \%$ of the lumen in the model group, and $22.49 \%$ in the blebbistatin group with significant difference (Fig. 1B \& C).

HE staining results showed that carotid artery endothelial surface integrity in the sham group was arranged in an orderly manner without thrombus formation in the lumen of the carotid artery or inflammatory cell infiltration (Fig. 1D). Carotid artery thrombosis was observed as compact red and white thrombus formation with thrombosis and the vascular wall connected to form a visible platelet trabecular. Most vascular endothelial cells exhibited shedding necrosis thrombosis with a vascular wall surrounded by inflammatory cell infiltration. Blebbistatin $(1 \mathrm{mg} / \mathrm{kg})$ treatment reduced the number of vascular endothelial cells at the surface and white blood cell adhesion and infiltration. Blebbistatin had no effect on the formation of carotid artery thrombosis in the sham group.

\section{Blebbistatin inhibited NMMHC IIA expression in the CAT model}

$\mathrm{IH}$ results showed that the carotid artery endothelium was intact with no NMMHC IIA expression in the sham-operated group (Fig. 2A \& B). NMMHC IIA expression in carotid artery vascular tissue increased significantly in the model group in the region relative to the average optical density value in the sham group. Blebbistatin reduced the damage in carotid artery vascular tissue, the NMMHC IIA expression level and the regional relative average optical density value. Furthermore, IF results indicated that no obvious NMMHC IIA expression (green) was observed in the sham group, and the carotid artery endothelium was intact (red) (Fig. 2C \& D). NMMHC IIA expression level in the carotid artery vascular tissue and endothelium was significantly higher, as verified by significantly enhanced fluorescence intensity (green and red and green overlap) in the model group compared to the sham operation group. Blebbistatin significantly reduced NMMHC IIA expression both in the carotid artery vascular tissue and endothelium. Blebbistatin had no obvious effect on the expression of NMMHC IIA in the sham group.

\section{Blebbistatin suppressed TF expression and activity in the CAT model}

$\mathrm{IH}$ results showed that the carotid artery endothelium was intact with no TF expression in the sham group (Fig. 3A \& B). TF expression levels were significantly elevated in the model group, which exhibited significantly increased average optical densities in relative regions. Blebbistatin decreased TF expression in the carotid artery vascular tissue and the value of the average optical density. Immunofluorescence revealed no TF expression (green) in carotid artery endothelium (red) in the sham group (Fig. 3C \& D), while TF signal was significantly increased (green) in the endothelium (red and green overlap) in the model group. Blebbistatin significantly decreased the fluorescence intensity of the TF signal in the carotid artery vascular tissue and the endothelium (red and green overlap). Meanwhile, ferric chloride markedly induced TF plasma procoagulant activity compared to the sham group with significant difference, while blebbistatin remarkably inhibited the elevation of plasma TF activity (Fig. 3E). Blebbistatin had no obvious effect on the expression and activation of TF in the sham group.

\section{Blebbistatin inhibited GSK3 $\beta$ expression and activation in the CAT model}

$\mathrm{IH}$ results showed that no obvious expression of GSK3 $\beta$ or $p-G S K 3 \beta$ was observed in the sham group. The expression level of carotid artery vascular tissue GSK3 $\beta$ or $p$-GSK3 $\beta$ and the relative value of the average optical density increased significantly in the model group. Blebbistatin reduced expression of GSK3 $\beta$ but increased the expression of $p-G S K 3 \beta$ in the carotid artery vascular tissue (Fig. 4A \& B and Fig. 5A \& B). 
A
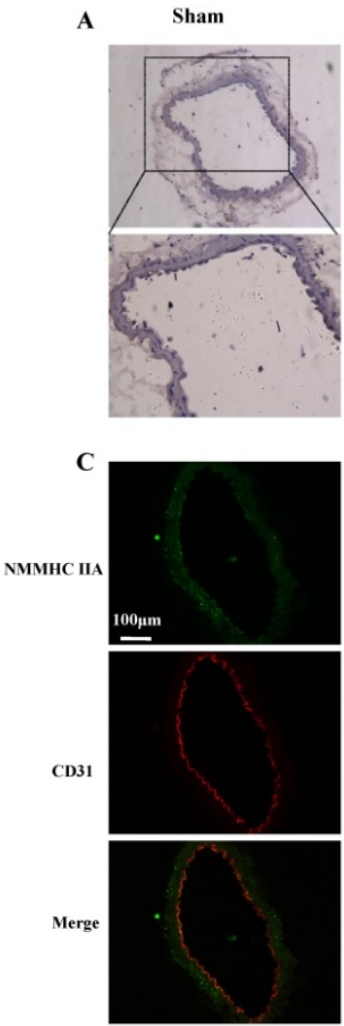

Model
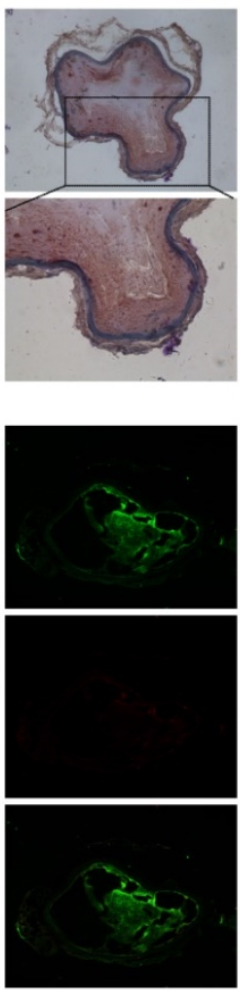

Model+Ble
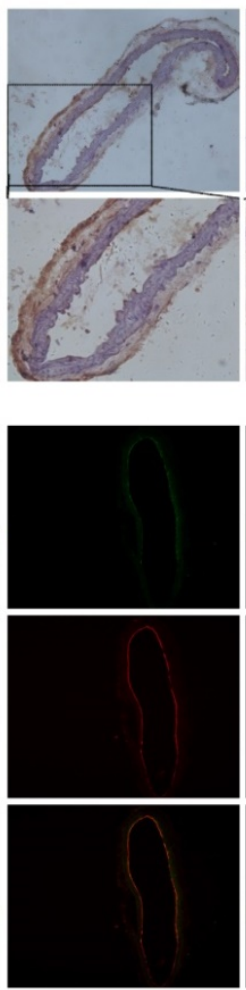

Sham+Ble
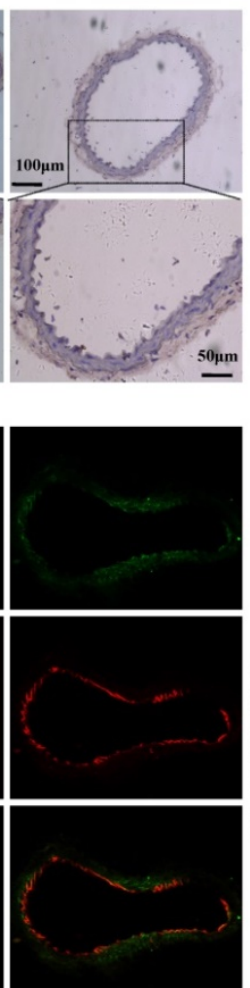

B

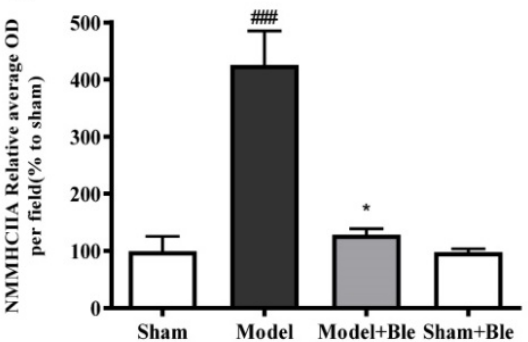

Figure 2. Blebbistatin inhibits NMMHC IIA expression in the CAT model. CAs were harvested for immunohistochemistry and immunofluorescence analyses $0.5 \mathrm{~h}$ after a 3 -minute application of $5 \%$ ferric chloride to the surface of the vessel. (A) \&(B). Representative photomicrographs demonstrating immunohistochemistry for NMMHC IIA in mouse carotid artery vascular tissues treated with: sham, sham+Blebbistatin, $5 \%$ ferric chloride, $5 \%$ ferric chloride+Blebbistatin. (C) \& (D). Sections were immunostained with a combination of anti-NMMHC IIA pAbs (green) and anti-CD31 pAbs (red). Images were digitally merged and fluorescence intensities were quantified using Image J software. Co-localization of NMMHC IIA and CD31 was quantified using Image Pro Plus software and calculated as a Pearson's correlation coefficient. Data represent means $\pm S E M(n=6)$. \#\#\#P<0.001 vs. sham group; $* * P<0.01$ vs. model group.

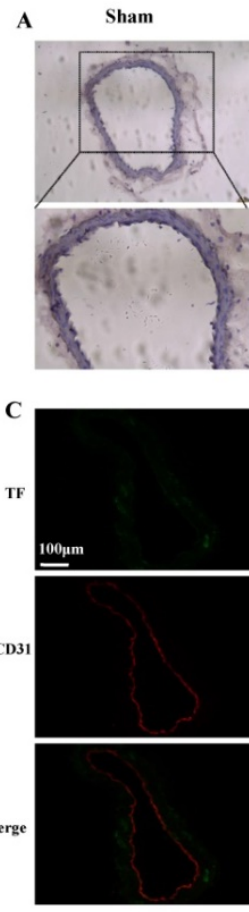

Sham
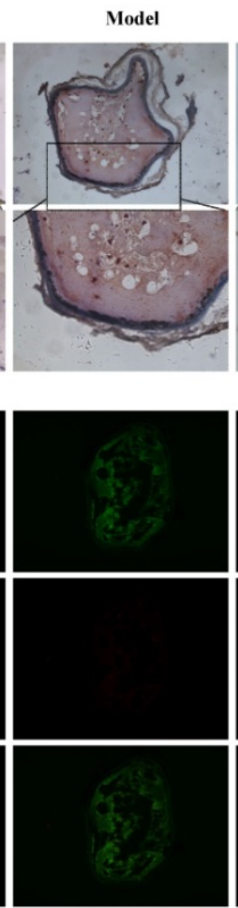

Model
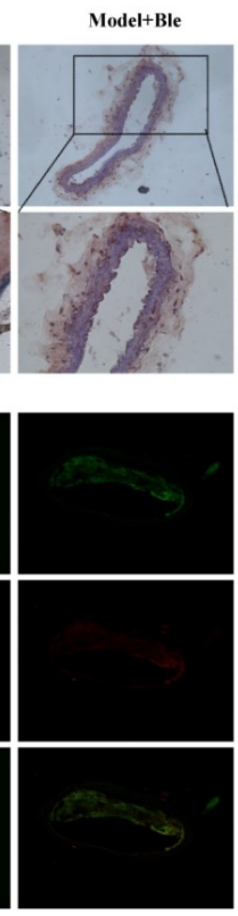

Model+Ble
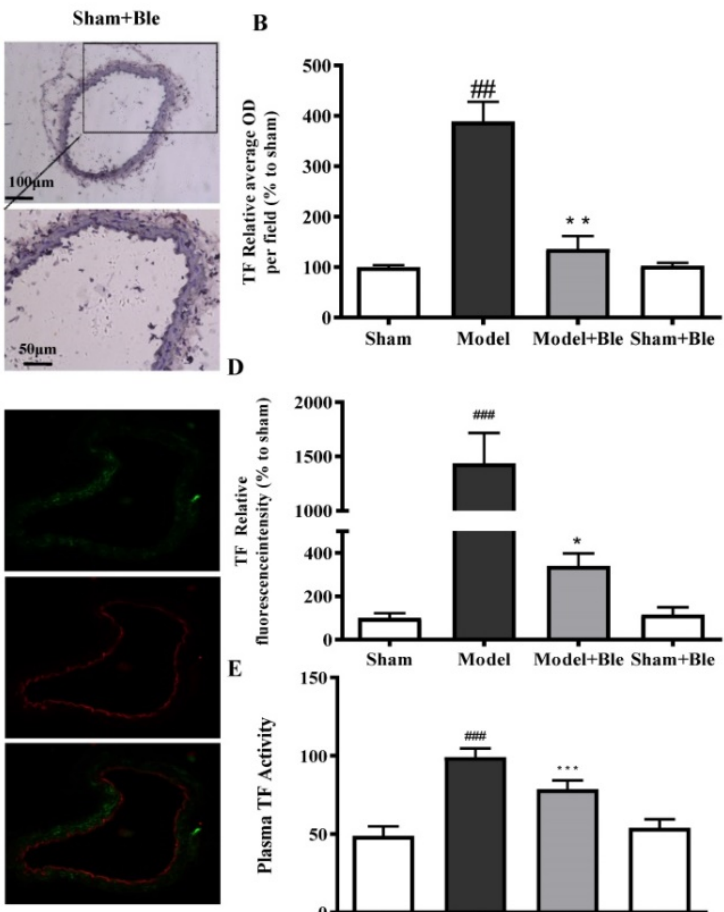

Sham+Ble

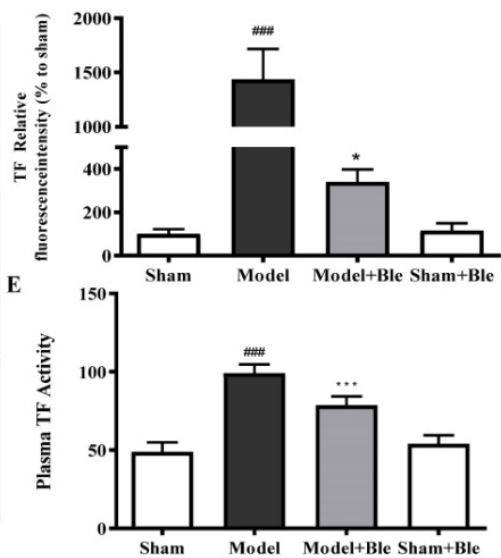

Figure 3. Blebbistatin inhibits TF expression and procoagulant activity in endothelium in the CAT model. Plasma was collected and used to determine TF procoagulant activity $0.5 \mathrm{~h}$ after a 3-minute application of $5 \%$ ferric chloride to the surface of the vessel. CAs were harvested for immunohistochemistry and immunofluorescence analysis. (A)\&(B). Representative photomicrographs demonstrating immunohistochemistry for TF in mouse carotid artery vascular tissues. (C)\&(D). CA sections were immunostained with a combination of anti-TF pAbs (green) and anti-CD31 pAbs (red). Images were digitally merged, and fluorescence intensity was quantified using Image J software. Co-localization of TF and CD31 was quantified using Image Pro Plus software and calculated as Pearson's correlation coefficients $(r)$. $(E)$. TF procoagulant activity was measured in mouse plasma. Data represent means $\pm S E M(n=6)$. \#\#\# $<0.001$ vs. sham group; $* * * \mathrm{P}<0.001$ vs. model group $* * \mathrm{P}<0.01$ vs. model group; $* \mathrm{P}<0.05$ vs. model group. 

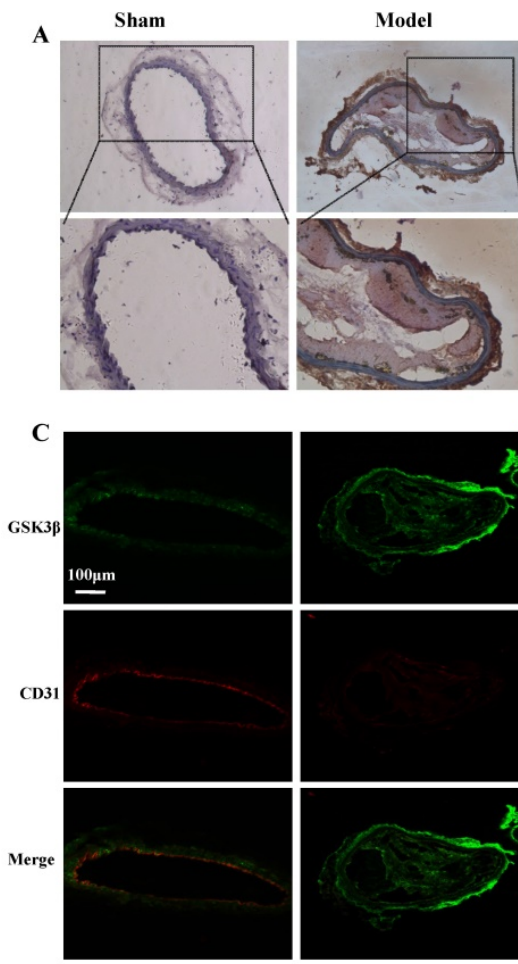

Sham

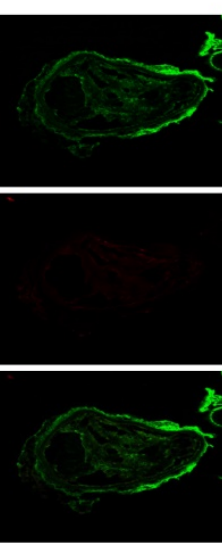

Model
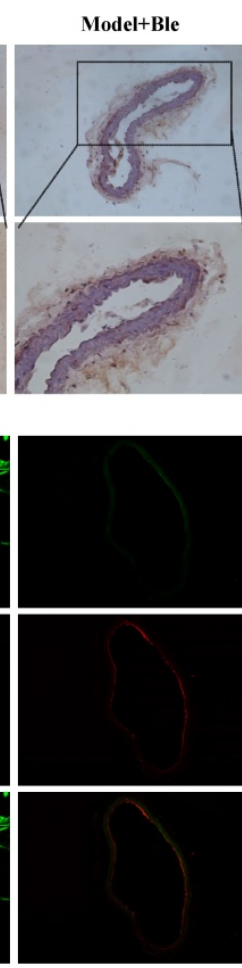

Model+Ble
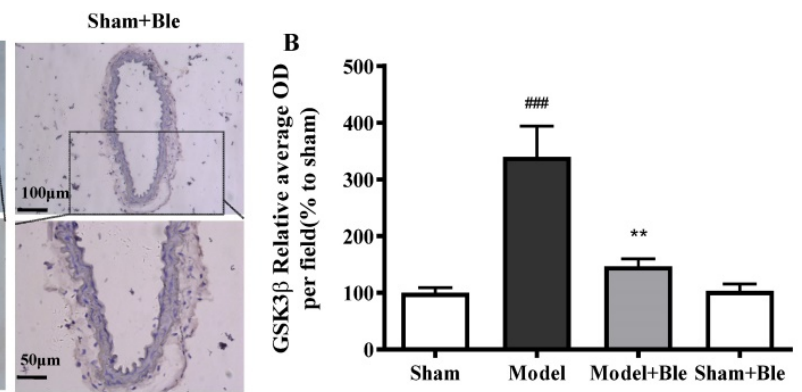

D

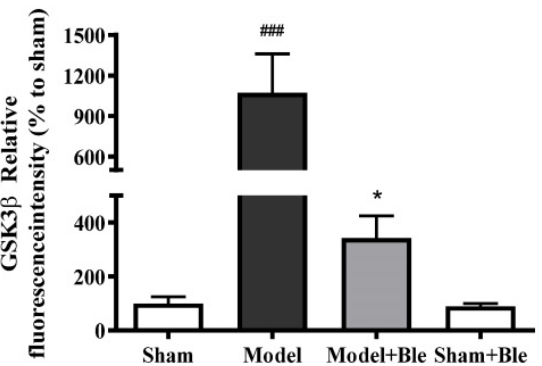

Figure 4. Blebbistatin inhibits GSK3 $\beta$ expression in the CAT model. CAs were harvested for immunohistochemistry and immunofluorescence analysis $0.5 \mathrm{~h}$ after a 3 -minute application of $5 \%$ ferric chloride to the surface of the vessel. (A)\&(B). Representative photomicrographs demonstrating immunohistochemistry for GSK $3 \beta$ in mouse carotid artery vascular tissues. (C)\&(D). CA sections were immunostained with a combination of anti-GSK3 $\beta$ pAbs (green) and anti-CD31 pAbs (red). Images were digitally merged, and fluorescence intensity was quantified using Image J software. Co-localization of GSK3 3 and CD31 was quantified using Image Pro Plus software and calculated as a Pearson's correlation coefficient ( $r$ ). Data represent means $\pm \operatorname{SEM}(\mathrm{n}=6)$. \#\#\# $<0.001$ vs. sham group; $* * \mathrm{P}<0.01$ vs. model group.
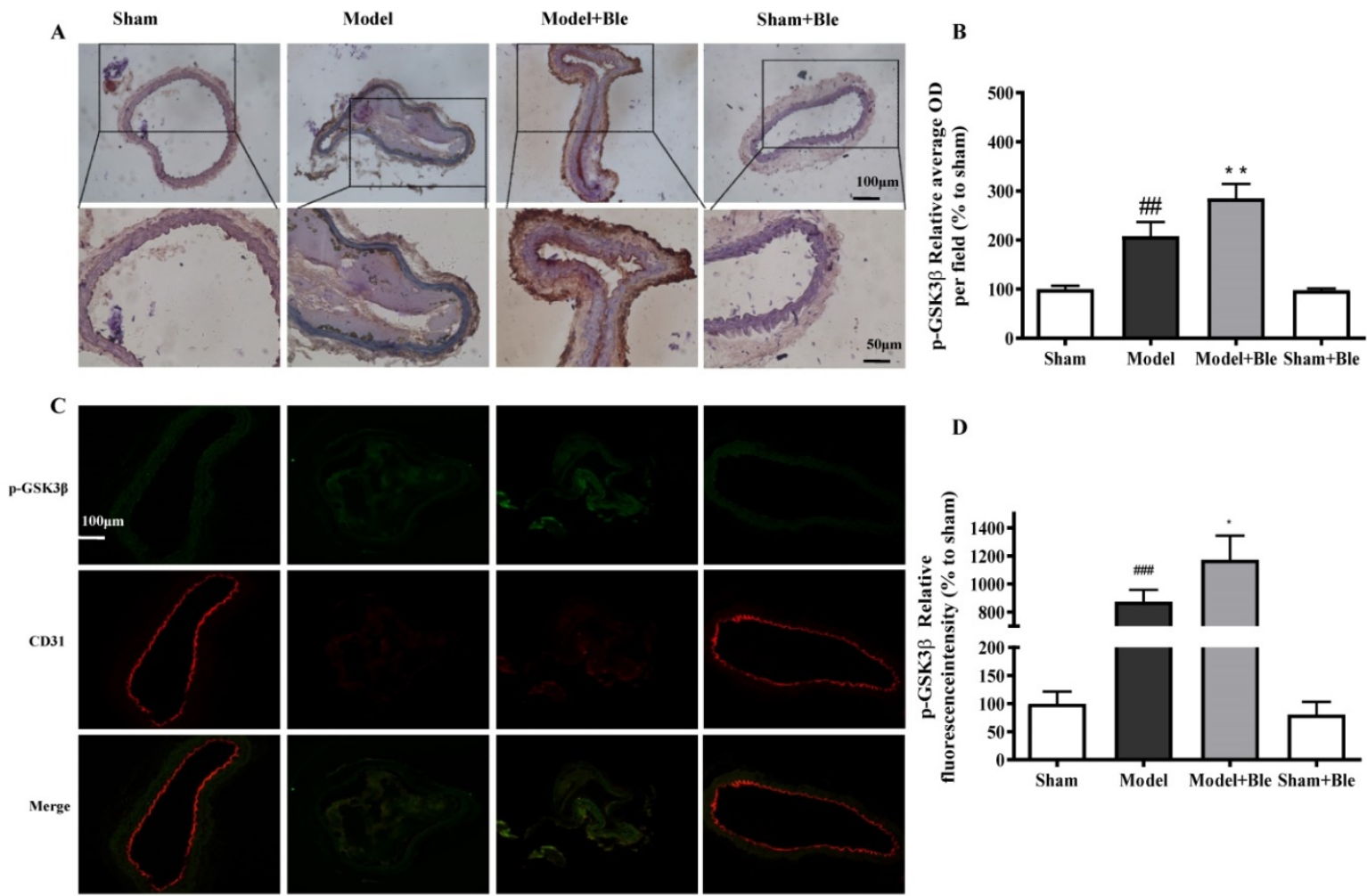

Figure 5. Blebbistatin inhibits p-GSK3 $\beta$ expression in the CAT model. CAs were harvested for immunohistochemistry and immunofluorescence analysis $0.5 \mathrm{~h}$ after a 3 -minute application of $5 \%$ ferric chloride to the surface of the vessel. (A)\&(B). Representative photomicrographs demonstrating immunohistochemistry for $p$-GSK $3 \beta$ in mouse carotid artery vascular tissues. (C)\&(D). CA sections were immunostained with a combination of anti-p-GSK3 $\beta$ pAbs (green) and anti-CD31 pAbs (red). Images were digitally merged, and fluorescence intensity was quantified using Image J software. Co-localization of $p-G S K 3 \beta$ and CD31 was quantified using Image Pro Plus software and calculated as a Pearson's correlation coefficient ( $r$ ). Data represent means $\pm \operatorname{SEM}(\mathrm{n}=6)$. \#\#\# $<0.001$ vs. sham group; $* * \mathrm{P}<0.01$ vs. model group. 
As shown in Fig. 4C \& D and Fig. 5C \& D, no obvious GSK3 $\beta$ or p-GSK3 $\beta$ (green) was observed in the sham group. The intensity of GSK3 $\beta$ or p-GSK3 $\beta$ in the carotid artery thrombosis group increased significantly. Blebbistatin significantly reduced the fluorescence intensity of GSK3 $\beta$ but increased the intensity of $p$-GSK3 $\beta$ in both the carotid artery vascular tissue (green) and endothelium (red and green overlap). Blebbistatin had no obvious effect on the expression of GSK3 $\beta$ and p-GSK3 $\beta$ in the sham group.

\section{Blebbistatin inhibited p65 expression and activation in the CAT model}

No obvious p65 or p-p65 signal was observed in the sham group. The expression of p65 or p-p65 and their relative average optical density values in carotid artery vascular tissue increased significantly in the model group. Blebbistatin significantly reduced the expression of p65 and p-p65 in the damaged carotid artery vascular tissue regions (Fig. 6A \& B and Fig. 7A \& B).
The carotid artery endothelium was intact, and no obvious p65 or p-p65 (green) expression was observed in the sham group. The expression of p65 and regional relative average optical density value increased significantly in the model group. Blebbistatin significantly reduced the expression of p65 or p-p65 in the carotid artery vascular tissue and endothelium (Fig. 6C \& D and Fig. 7C \& D). Blebbistatin had no obvious effect on the expression of p65 and p-p65 in the sham group.

\section{Discussion}

Based on previous studies, we further explored the function of NMMHC IIA in artery thrombosis. This study confirmed that NMMHC IIA expression was increased in $\mathrm{FeCl}_{3}$-induced carotid artery thrombosis, and blebbistatin inhibited NMMHC IIA expression and plasma $\mathrm{TF}$, promoted coagulation activity and suppressed thrombosis. These results corroborated the role of NMMHC IIA-mediated TF.
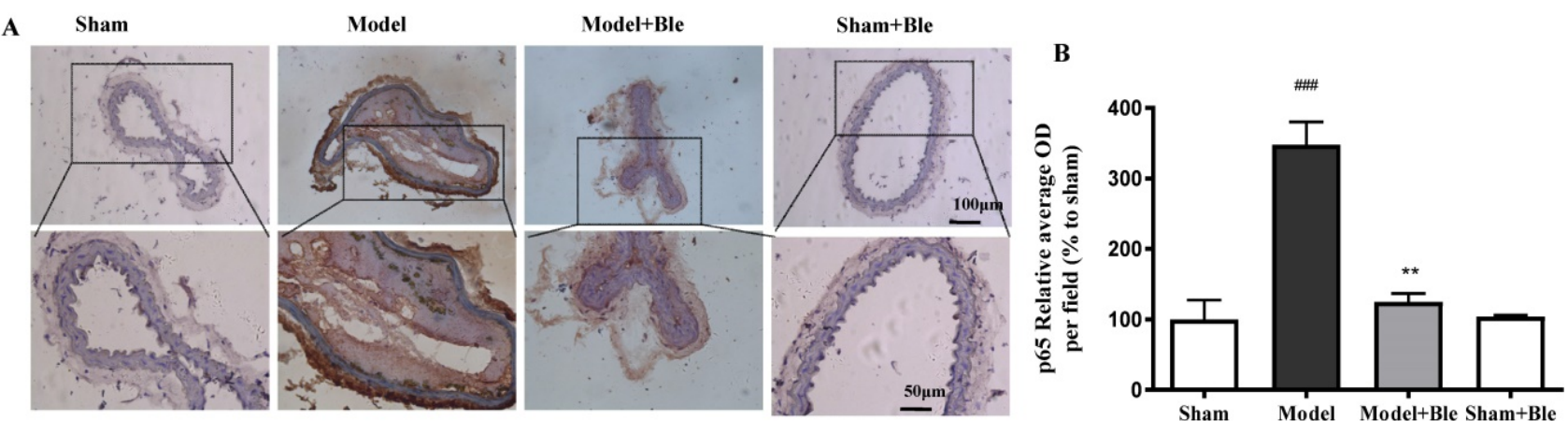

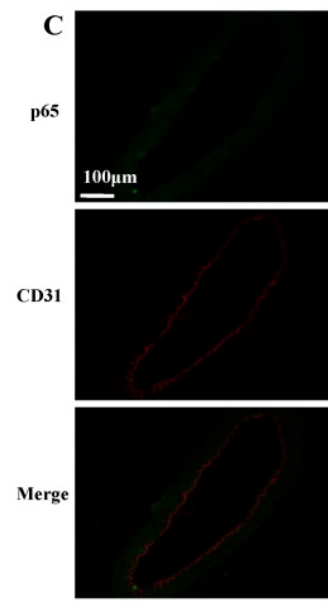

Sham

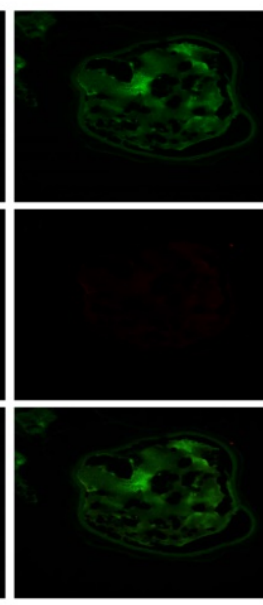

Model

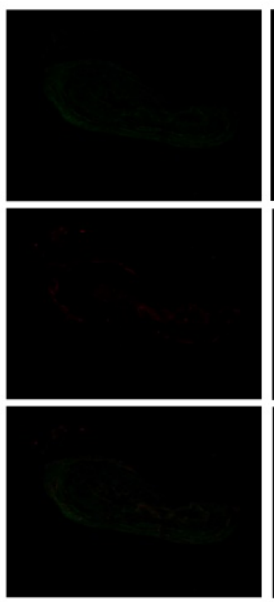

Model+Ble

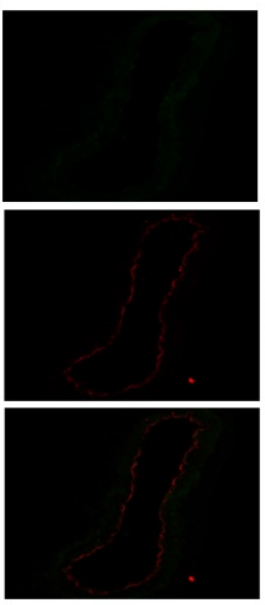

Sham+Ble

Figure 6. Blebbistatin inhibits $\mathbf{p} 65$ expression in the CAT model. CAs were harvested for immunohistochemistry and immunofluorescence analysis $0.5 \mathrm{~h}$ after a 3 -minute application of $5 \%$ ferric chloride to the surface of the vessel. (A)\&(B). Representative photomicrographs demonstrating immunohistochemistry for p65 in mouse carotid artery vascular tissues. (C)\&(D). CA sections were immunostained with a combination of anti-p65 pAbs (green) and anti-CD31 pAbs (red). Images were digitally merged, and fluorescence intensity was quantified using Image J software. Co-localization of $\mathrm{p} 65$ and CD31 was quantified using Image Pro Plus software and calculated as a Pearson's correlation coefficient $(r)$. Data represent means $\pm S E M$ ( $n=6$ ). $\# \# \#<0.001$ vs. sham group; ${ }^{* * P}<0.01$ vs. model group. 
A
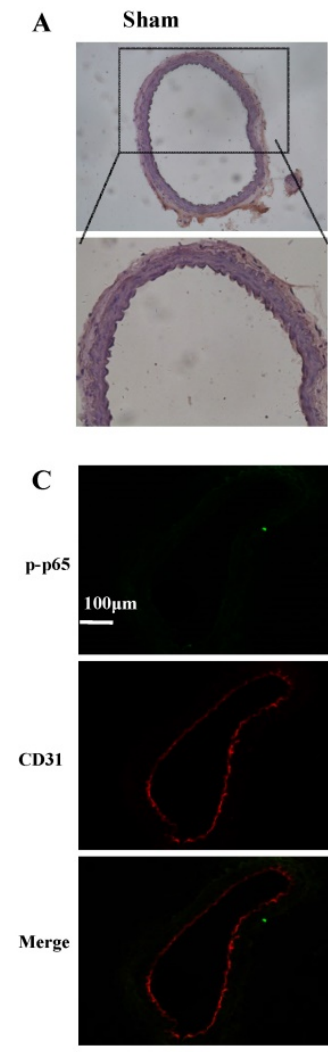

Sham
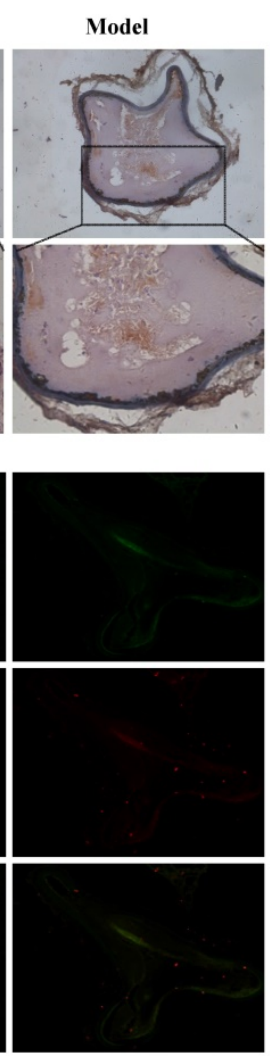

Model
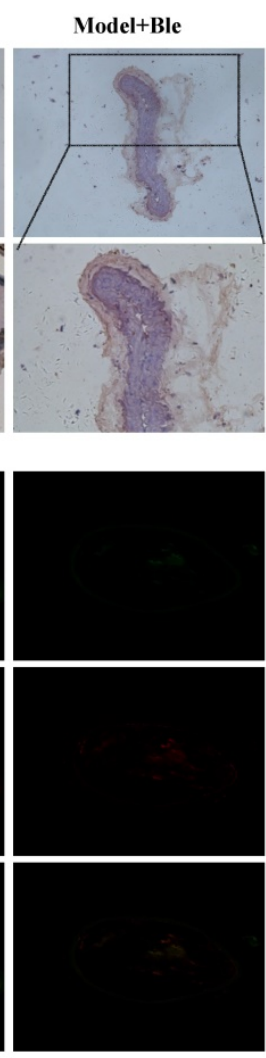

Model+Ble
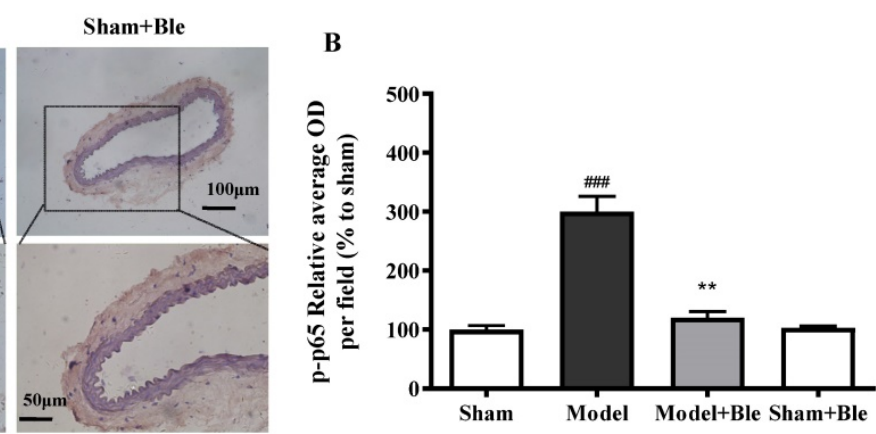

D
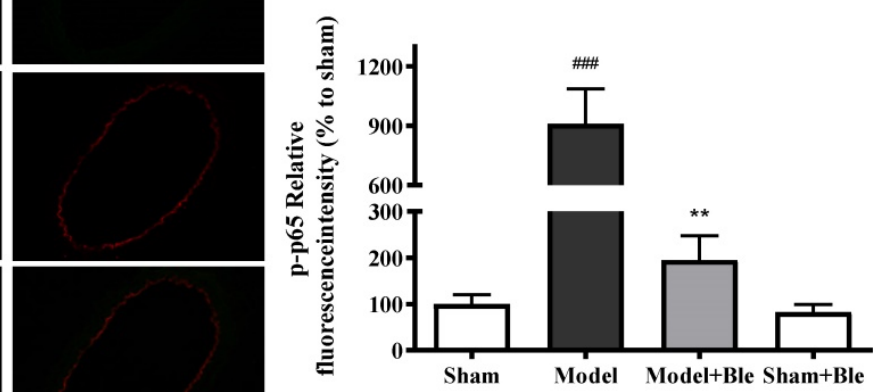

Sham Model Model+Ble Sham+Ble

Figure 7. Blebbistatin inhibits p-p65 expression in the CAT model.CAs were harvested for immunohistochemistry and immunofluorescence analysis $0.5 \mathrm{~h}$ after a 3 -minute application of $5 \%$ ferric chloride to the surface of the vessel. (A)\&(B). Representative photomicrographs demonstrating immunohistochemistry for $p$-p65 in mouse carotid artery vascular tissues. (C)\&(D). $C A$ sections were immunostained with a combination of anti-p-p 65 pAbs (green) and anti-CD 31 pAbs (red). Images were digitally merged, and fluorescence intensity was quantified using Image $\mathrm{J}$ software. Co-localization of $\mathrm{p}-\mathrm{p} 65$ and CD31 was quantified using Image Pro Plus software and calculated as a Pearson's correlation coefficient $(r)$. Data represent means $\pm S E M(n=6)$. \#\#P<0.001 vs. sham group; ${ }^{* * P}<0.01$ vs. model group.

Despite much research, the pathological mechanisms of AT are not completely clear, and there is a lack of clinically safe and effective drug treatments. Numerous studies have demonstrated a relationship between atherosclerosis and arterial thrombosis, and vascular endothelial injury caused by inflammation is the primary factor that initiates atherosclerosis and thrombosis [22-24]. Vascular endothelial cells inhibit platelet aggregation, stimulate fibrinolysis and inhibit the physiological function of abnormal coagulation under normal physiological conditions. Activation of vascular endothelial cells stimulates platelet aggregation and the inhibition of fibrin dissolution, which contributes to abnormal luminal coagulation and leads to thrombus formation [24]. Therefore, it is beneficial to prevent and cure AT by effectively regulating endothelial function, reducing the inflammatory reaction and improving the coagulation balance.

The pathophysiological changes and the evaluation of various types of anti-thrombotic drugs and treatment methods have become one of the important methods in the basic medical research to study the mechanism of thrombosis in vivo. $\mathrm{FeCl}_{3}$ was used in this study to induced carotid artery thrombosis. This model leads to severe endothelial cell injury induced by oxidative stress and enhances the expression of TF in endothelial cells and leukocytes [25]. Thrombus formation was close to the clinical spontaneous thrombosis and tissue morphology and rich in thrombi, fibrin and red blood cells. Therefore, this model has been widely used in preclinical evaluation of thrombolytic drugs and research [26]. Recent studies have demonstrated that conditional deficiency of NMMHC IIA in platelets reduces platelet aggregation and arterial thrombosis induced by $\mathrm{FeCl}_{3}[16,17]$, and the NMMHC IIA inhibitor blebbistatin inhibited expression of NMMHC IIA and TF to reduce deep vein thrombosis [18]. Furthermore, blebbistatin has a direct anti-inflammatory effect [27]. However, there are no reports of whether blebbistatin could inhibit arterial thrombosis and modulate TF-related signalling pathways in arterial vascular tissues or endothelium. In the present study, we have demonstrated that blebbistatin improved the blood flow value of the carotid artery and alleviated thrombosis and internal carotid lumen-filling material, which gave the first 
evidence that blebbistatin could inhibit artery thrombosis (Shown in Fig. 1A-D). With the help of a Doppler flow probe, it was indicated that blebbistatin ( $1 \mathrm{mg} / \mathrm{kg}$ ) improved the blood flow value of the carotid artery compared to the model group. As shown with HE staining and the thrombosis weight statistics, blebbistatin could alleviate thrombosis and internal carotid lumen-filling material.

As reported, the expression of NMMHC IIA increased in the endothelium in the venous thrombosis model [18]. However, there was no report on the function of NMMHC IIA in the AT model. In this study, we have demonstrated that the vascular and endothelial expression of NMMHC IIA increased in $\mathrm{FeCl}_{3}$-induced carotid artery thrombosis, and blebbistatin inhibited NMMHC IIA expression in the carotid artery vascular tissue injury group (Fig. 2). This result indicated that NMMHC IIA played an important role in the AT model.

As the first promoter of the coagulation cascade under physiological conditions and the extrinsic coagulation pathway under pathological conditions, TF expression and activity is up-regulated in atherosclerosis, deep vein thrombosis, pulmonary embolism, and angina; it is disseminated in blood coagulation, hypertension, cancer and many other diseases [28-31]. Therefore, intervention with the TF pathway is an important strategy for the prevention and treatment of thrombotic diseases [32, 33]. As reported, the deficiency of TLR-4 could reduce TF expression and activity and extend the thrombosis formation time in $\mathrm{FeCl}_{3}$-induced carotid artery injury [25]. It was found that the NMMHCIIA non-specific inhibitor blebbistatin reduced TF expression in the endothelium in vitro and inhibited deep vein thrombosis formation in vivo [18]. However, the regulation of TF expression in endothelial cells is not completely clear, and there are no drugs for the prevention and treatment of thrombosis related to the modulation of these important functional proteins. This paper confirmed that TF expression and activity increased in the $\mathrm{FeCl}_{3}$-treated group, and the NMMHC IIA inhibitor blebbistatin reduced TF expression and activity (Shown in Fig. 3). This result was consistent with existing reports.

Nuclear factor (NF)- $\mathrm{kB}$ and phosphoinositide 3-kinase (PI3K) primarily regulate TF expression in response to inflammatory stimuli $[12,34]$. The modulation of these inter-related signalling pathways and inhibition of TF expression or activity may exert therapeutic effects against venous thrombosis. The NF-KB signalling pathway regulates TF expression, and the PI3K/Akt signalling pathway negatively regulates $\mathrm{TF}$ expression primarily by promoting GSK3 $\beta$ (activated form) phosphorylation [35-37]. As reported, GSK3 $\beta$, which is a ser-thr kinase that is phosphorylated by the kinase Akt, acted as a negative regulator of platelet function in vitro and in vivo [38]. AZD484, a selective PI3K $\beta$ inhibitor, can make a p110beta deletion in mouse platelets. Within the growing platelet thrombus, p110beta inactivation impairs the activating phosphorylation of Akt and the inhibitory phosphorylation of GSK3. GSK3 $\beta$ was activated in the endothelium in the venous thrombosis model [18]. In accordance with these data, pharmacologic inhibition of GSK3 restores thrombus stability [39]. This study examined blebbistatin treatment in a $\mathrm{FeCl}_{3}$-induced mouse model of carotid artery thrombosis and GSK3 $\beta$ and NF-KB signalling pathway activity. This paper confirmed for the first time that GSK3 $\beta$ was activated in the $\mathrm{FeCl}_{3}$-induced AT model. The results demonstrated for the first time that blebbistatin activated the phosphorylation of GSK3 $\beta$ via the PI3K/Akt pathway through inhibition of GSK3 $\beta$ expression in vascular tissue (Fig. 4 \& 5). These results first demonstrated that blebbistatin significantly activates the GSK3 $\beta$-related signalling pathway to inhibit TF expression, and its mechanism may be a negative regulatory mechanism. The activation of GSK3 $\beta$ is an inactive form, which negatively regulates the inhibition of NF- $\mathrm{KB}$ activity. Blebbistatin inhibited the activity and expression of key proteins in the NF-KB pathway, phospho-p65 and p65, in carotid artery vascular endothelial tissue (Fig. $6 \& 7)$.

Our results support the development of drugs targeting NMMHC IIA for the prevention and treatment of thrombotic diseases. However, the NMMHC IIA inhibitor blebbistatin exhibits severe phototoxicity, and it is not suitable for drug development $[40,41]$. The development of a highly efficient, safe, non-toxic NMMHC IIA inhibitor for the treatment of thrombotic disease is urgently needed. In addition, blebbistatin suppresses both NMMHC IIA function and the NMMHC IIA expression in vivo, suggesting that conditional NMMHC IIA-KO in endothelial cells may contribute to prevent AT in mice, which requires further study. Moreover, endothelial cells played an important role in the process of AT. The expression of NMMHC IIA, TF, GSK3 $\beta$ and p65 changed in the endothelium. However, the expression of these proteins also changed in the vascular cells. Meanwhile, there are many other cells involved in AT, such as smooth muscle cells and macrophages. Whether other cells participate in the blebbistatin inhibition of AT needs further research. There was only an in vivo study in this research; more study is needed to understand the mechanism of blebbistatin on the inhibition of AT.

These results confirmed that blebbistatin 
inhibited NMMHC IIA function and expression via the GSK3 $\beta-N F-\kappa B$ signalling pathway and the down-regulation of TF expression and activity in endothelium. Blebbistatin had no obvious effect on the formation of AT and the expression of related signalling pathway components. These results provide compelling evidence of the role of NMMHC IIA in AT and may lead to new strategies for the treatment of AT and many other inflammation-related thrombotic diseases.

\section{Acknowledgements}

This study was supported by the National
Natural Science Foundation of China (No.81274131\&No.81503295), 2011' Program for Excellent Scientific and Technological Innovation Team of Jiangsu Higher Education, a Project Funded by the Priority Academic Program Development of Jiangsu Higher Education Institutions, and Jiangsu Province 2011 Plan for Collaborative Innovation.

\section{Competing Interests}

The authors have declared that no competing interest exists.

\section{References}

1. Santarius T, Menon D.K. Images in clinical medicine. Carotid-artery thrombosis secondary to basal skull fracture. N Engl J Med.2003;349:e5.

2. Stoll G, Kleinschnitz C, Nieswandt B. Molecular mechanisms of thrombus formation in ischemic stroke: novel insights and targets for treatment. Blood. 2008; 112:3555-3562.

3. Keenan NG, Sheppard MN, Nott DM, et al. Carotid plaque rupture. Lancet.2009;374:1703.

4. Blanco M, Sobrino T, Montaner J, et al. Stroke with polyvascular atherothrombotic disease. Atherosclerosis. 2010;208:587-592.

5. Roche P, Ritzenthaler $\mathrm{T}$, Nighoghossian N. Cerebral venous thrombosis causing simultaneous pulmonary embolism and internal carotid artery stroke. Rev Neurol (Paris).2016; 172:167-168.

6. Kanemaru K, Nishiyama Y, Yoshioka $H$, et al. In-stent thrombosis after carotid artery stenting despite sufficient antiplatelet therapy in a bladder cancer patient. J Stroke Cerebrovasc Dis.2013;22:1196-1200.

7. Viaccoz A, Muller H, Perren F. Asymptomatic external carotid artery thrombosis in acute stroke. Ultraschall Med. 2012·33·E357-358.

8. Aue G, Nelson Lozier J, Tian X, et al. Inflammation, TNFalpha and endothelial dysfunction link lenalidomide to venous thrombosis in chronic lymphocytic leukemia. Am J Hematol. 2011;86:835-840.

9. Stampfli SF, Akhmedov A, Gebhard C, et al. Aging induces endothelial dysfunction while sparing arterial thrombosis. Arterioscler Thromb Vasc Biol.2010;30:1960-1967.

10. Ruf W. Role of thiol pathways in TF procoagulant regulation. Thromb Res.2012;129 Suppl 2:S11-12.

11. Li S, Chen H, Ren J, et al. MicroRNA-223 inhibits tissue factor expression in vascular endothelial cells. Atherosclerosis.2014;237:514-520

12. Dong R, Chen W. Feng W, et al. Exogenous Bradykinin Inhibits Tissue Factor Induction and Deep Vein Thrombosis via Activating the eNOS/Phosphoinositide 3-Kinase/Akt Signaling Pathway. Cell Physiol Biochem.2015;37:1592-1606.

13. Winckers $\mathrm{K}$, ten Cate $\mathrm{H}$, Hackeng TM. The role of tissue factor pathway inhibitor in atherosclerosis and arterial thrombosis. Blood Rev.2013;27:119-132.

14. Juanes-Garcia A, Llorente-Gonzalez C, Vicente-Manzanares M. Molecular control of non-muscle myosin II assembly. Oncotarget. 2016;7:5092-5093.

15. Kunishima S, Yoshinari M, Nishio $\mathrm{H}$, et al. Haematological characteristics of MYH9 disorders due to MYH9 R702 mutations. Eur J Haematol.2007;78:220-226.

16. Eckly A, Strassel C, Freund M, et al. Abnormal megakaryocyte morphology and proplatelet formation in mice with megakaryocyte-restricted MYH9 inactivation. Blood.2009;113:3182-189.
17. Leon C, Eckly A, Hechler B, et al. Megakaryocyte-restricted MYH9 inactivation dramatically affects hemostasis while preserving platelet aggregation and secretion. Blood. 2007;110:3183-3191.

18. Zhai KF, Tang YM, Zhang YY, et al. NMMHC IIA inhibition impedes tissue factor expression and venous thrombosis via Akt/GSK3beta-NF-kappaB signalling pathways in the endothelium. Thromb Haemost. 2015;114:173-185.

19. Kurz KD, Main BW, Sandusky GE. Rat model of arterial thrombosis induced by ferric chloride. Thromb Res.1990;60:269-280.

20. Robinson MA, Welsh DC, Bickel DJ, et al. Differential effects of sodium nitroprusside and hydralazine in a rat model of topical $\mathrm{FeCl} 3$-induced carotid artery thrombosis. Thromb Res.2003;111:59-64.

21. van den Hengel LG, Osanto S, Reitsma PH, et al. Murine tissue factor coagulant activity is critically dependent on the presence of an intact allosteric disulfide. Haematologica.2013;98:153-158.

22. Furie B, Furie BC. Mechanisms of thrombus formation. N Engl J Med. 2008;359:938-949.

23. Lu J, Xiang G, Liu M, et al. Irisin protects against endothelial injury and ameliorates atherosclerosis in apolipoprotein E-Null diabetic mice. Atherosclerosis. 2015;243:438-448.

24. Yau JW., Teoh H, Verma S. Endothelial cell control of thrombosis. BMC Cardiovasc Disord.2015;15:130.

25. Ren M, Li R, Luo M, et al. Endothelial cells but not platelets are the major source of Toll-like receptor 4 in the arterial thrombosis and tissue factor expression in mice. Am J Physiol Regul Integr Comp Physiol.2014;307:R901-907.

26. Neeves KB. Physiochemical artifacts in $\mathrm{FeCl} 3$ thrombosis models. Blood.2015; 126:700-701

27. Si J, Ge Y, Zhuang S, et al. Inhibiting nonmuscle myosin II impedes inflammatory infiltration and ameliorates progressive renal disease. Lab Invest.2010;90:448-458.

28. Luyendyk JP, Schabbauer GA, Tencati M, et al. Genetic analysis of the role of the PI3K-Akt pathway in lipopolysaccharide-induced cytokine and tissue factor gene expression in monocytes/macrophages. J Immunol. 2008; 180:4218-4226.

29. Mackman N. The many faces of tissue factor. J Thromb Haemost. 2009; 7 Suppl 1:136-139.

30. Kretz CA, Vaezzadeh N, Gross PL. Tissue factor and thrombosis models. Arterioscler Thromb Vasc Biol.2010; 30:900-908.

31. Tatsumi K, Ohashi K, Matsubara Y, et al. Tissue factor triggers procoagulation in transplanted mesenchymal stem cells leading to thromboembolism. Biochem Biophys Res Comm. 2013;431:203-209.

32. Steffel J, Luscher TF, Tanner FC. Tissue factor in cardiovascular diseases: molecular mechanisms and clinical implications. Circulation.2006; 113:722-731.

33. Holy EW, Tanner FC. Tissue factor in cardiovascular disease pathophysiology and pharmacological intervention. Adv Pharmacol. 2010;59:259-292.

34. Cirillo P, Ziviello F, Pellegrino G, et al. The adipokine apelin-13 induces expression of prothrombotic tissue factor. Thromb Haemost.2015;113:363-372.

35. Zhang $\mathrm{W}$, Wang $\mathrm{J}$, Wang $\mathrm{H}$, et al. Acadesine inhibits tissue factor induction and thrombus formation by activating the phosphoinositide 3-kinase/Akt signaling pathway. Arterioscler Thromb Vasc Biol.2010; 30:1000-1006.

36. Schabbauer G, Tencati M, Pedersen B, et al. PI3K-Akt pathway suppresses coagulation and inflammation in endotoxemic mice. Arterioscler Thromb Vasc Biol.2004;24:1963-1969.

37. Guha M, Mackman N. The phosphatidylinositol 3-kinase-Akt pathway limits lipopolysaccharide activation of signaling pathways and expression of inflammatory mediators in human monocytic cells. J Biol Chem.2002; 277:32124-32132.

38. Li D, August S, Woulfe DS. GSK3beta is a negative regulator of platelet function and thrombosis. Blood. 2008;111:3522-3530.

39. Laurent PA, Severin S, Hechler B, et al. Platelet PI3Kbeta and GSK3 regulate thrombus stability at a high shear rate. Blood.2015;125:881-888.

40. Swift LM, Asfour H, Posnack NG, et al. Properties of blebbistatin for cardiac optical mapping and other imaging applications. Pflugers Arch.2012; 464:503-512.

41. Kolega J. Phototoxicity and photoinactivation of blebbistatin in UV and visible light. Biochem Biophys Res Commun.2004;320:1020-1025. 